AperTO - Archivio Istituzionale Open Access dell'Università di Torino

\title{
Central binomial coefficients divisible by or coprime to their indices
}

\section{This is the author's manuscript}

Original Citation:

Availability:

This version is available http://hdl.handle.net/2318/1651940

since 2017-11-16T11:52:43Z

Published version:

DOI:10.1142/S1793042118500707

Terms of use:

Open Access

Anyone can freely access the full text of works made available as "Open Access". Works made available under a Creative Commons license can be used according to the terms and conditions of said license. Use of all other works requires consent of the right holder (author or publisher) if not exempted from copyright protection by the applicable law. 


\title{
CENTRAL BINOMIAL COEFFICIENTS DIVISIBLE BY OR COPRIME TO THEIR INDICES
}

\author{
CARLO SANNA
}

\begin{abstract}
Let $\mathcal{A}$ be the set of all positive integers $n$ such that $n$ divides the central binomial coefficient $\left(\begin{array}{c}2 n \\ n\end{array}\right)$. Pomerance proved that the upper density of $\mathcal{A}$ is at most $1-\log 2$. We improve this bound to $1-\log 2-0.05551$. Moreover, let $\mathcal{B}$ be the set of all positive integers $n$ such that $n$ and $\left(\begin{array}{c}2 n \\ n\end{array}\right)$ are relatively prime. We show that $\#(\mathcal{B} \cap[1, x]) \ll x / \sqrt{\log x}$ for all $x>1$.
\end{abstract}

\section{INTRODUCTION}

Given a sequence of integers $\left(a_{n}\right)_{n \geq 1}$ with some combinatorial or number-theoretic meaning, the study of the set of positive integers $n$ such that $n$ divides $a_{n}$ has interested several authors. For instance, Alba González, Luca, Pomerance, and Shparlinski [1] considered the case of $\left(a_{n}\right)_{n \geq 1}$ being a linear recurrence; while André-Jeannin [2], Luca and Tron [5], Sanna [7], and Somer [10] focused on Lucas sequences. Furthermore, Chen, Gassert and Stange [3] studied this problem for sequences given by the iterates of a polynomial map. Also, the "dual" set of positive integers $n$ such that $a_{n}$ and $n$ are relatively prime has been studied by Sanna [8] for $\left(a_{n}\right)_{n \geq 1}$ being a linear recurrence.

Let $\mathcal{A}$ be the set of positive integers $n$ such that $n$ divides the central binomial coefficient $\left(\begin{array}{c}2 n \\ n\end{array}\right)$. Ulas and Schinzel [12, Theorems 3.2 and 3.4] proved that $\mathcal{A}$ and its complement $\mathbb{N} \backslash \mathcal{A}$ are both infinite. We recall that the upper density of a set of positive integers $\mathcal{S}$ is defined as the value of

$$
\limsup _{x \rightarrow+\infty} \frac{\# \mathcal{S}(x)}{x}
$$

where $\mathcal{S}(x):=\mathcal{S} \cap[1, x]$, while the lower density is defined analogously by a limit inferior.

Pomerance [6, Theorem. 3] proved the following result.

Theorem 1.1. The upper density of $\mathcal{A}$ is at most $1-\log 2=0.30685 \ldots$

Note that Pomerance, maybe for aesthetic reasons, stated Theorem 1.1 with $1 / 3$ instead of $1-\log 2$, but he actually proved the latter bound.

Our first result is the following improvement of Theorem 1.1.

Theorem 1.2. The upper density of $\mathcal{A}$ is at most $1-\log 2-0.05551=0.25134 \ldots$

Let $\mathcal{B}$ be the set of all positive integers $n$ such that $\left(\begin{array}{c}2 n \\ n\end{array}\right)$ and $n$ are relatively prime. It is easy to see that each odd prime number belongs to $\mathcal{B}$. Hence, by the Prime Number Theorem, we have

$$
\# \mathcal{B}(x) \geq(1+o(1)) \cdot \frac{x}{\log x},
$$

as $x \rightarrow+\infty$. Our second result is an upper bound for $\# \mathcal{B}(x)$.

Theorem 1.3. We have

$$
\# \mathcal{B}(x) \ll \frac{x}{\sqrt{\log x}}
$$

for all $x>1$.

2010 Mathematics Subject Classification. Primary: 11N25. Secondary: 11A07, 11B65.

Key words and phrases. central binomial coefficient; divisibility; upper and lower densities. 
We leave the following questions to the interested readers:

Question 1.1. Does $\mathcal{A}$ have a positive lower density?

Question 1.2 . If $\beta(x)$ is defined implicitly by

$$
\# \mathcal{B}(x)=\frac{x}{(\log x)^{\beta(x)}},
$$

for all $x>1$, then from (1) and (2) it follows that

$$
\liminf _{x \rightarrow+\infty} \beta(x) \geq \frac{1}{2} \quad \text { and } \quad \limsup _{x \rightarrow+\infty} \beta(x) \leq 1 .
$$

Can we improved these bounds?

Pomerance [6, end of pag. 7] conjectured that Question 1.1 has a positive answer, and indeed numerical experiments [9] seem to suggest that the lower density of $\mathcal{A}$ is at least $1 / 9$.

Notation. We use the Landau-Bachmann "Big Oh" and "little oh" notations $O$ and $o$, as well as the Vinogradov symbol «. In summation subscripts, we reserve the letters $p$ and $q$ for prime numbers. For $x>0$, we write $\pi(x)$ for the number of primes not exceeding $x$. Finally, for integers $a$ and $m>0$, we use $(a \bmod m)$ to denote the unique nonnegative integer $r<m$ such that $m$ divides $a-r$.

\section{Preliminaries}

The key tool of the proofs is the following lemma.

Lemma 2.1. For all prime numbers $p$ and all positive integers $n$, we have that $p$ does not divide $\left(\begin{array}{c}2 n \\ n\end{array}\right)$ if and only if all the digits of $n$ written in base $p$ are less that $p / 2$.

Proof. It is a corollary of the theorem of Kummer [4] which says that, for positive integers $m, n$ and a prime number $p$, the exponent of $p$ in the prime factorization of $\left(\begin{array}{c}m+n \\ n\end{array}\right)$ is equal to the number of carries in the addition $m+n$ when done in base $p$.

The next lemma follows easily from Lemma 2.1 by a counting argument.

Lemma 2.2. For all prime numbers $p$ and all $x \geq 2$, the number of positive integers $n \leq x$ such that $p$ does not divide $\left(\begin{array}{c}2 n \\ n\end{array}\right)$ is at most $p x^{\theta_{p}}$, where $\theta_{p}:=\log \left(\frac{1}{2}(p+1)\right) / \log p$.

Proof. See [6, Lemma 1].

We need also the following well-known theorem of Mertens.

Theorem 2.3. We have

$$
\sum_{p \leq x} \frac{1}{p}=\log \log x+M+O\left(\frac{1}{\log x}\right),
$$

for all $x \geq 2$, where $M$ is the Meissel-Mertens constant.

Proof. See [11, Chapter I.1, Theorem. 9].

A consequence of Theoreom 2.3 is the following technical lemma.

Lemma 2.4. We have

$$
\sum_{\substack{\sqrt[3]{2 x}<p \leq \sqrt{2 x} \\ \sqrt{2 x}<q \leq x / p}} \frac{1}{p q}<0.06502+o(1)
$$

as $x \rightarrow+\infty$. 
Proof. Fix a positive integer $n$ and, for the sake of convenience, put $\alpha_{k}:=1 / 3+k /(6 n)$ for each nonnegative integer $k \leq n$. Thanks to Theorem 2.3, we have

$$
\begin{aligned}
\sum_{\substack{\sqrt[3]{2 x}<p \leq \sqrt{2 x} \\
\sqrt{2 x}<q \leq x / p}} \frac{1}{p q} & =\sum_{k=1}^{n} \sum_{(2 x)^{\alpha_{k-1}<p \leq(2 x)^{\alpha_{k}}}} \frac{1}{p} \sum_{\sqrt{2 x}<q \leq x / p} \frac{1}{q} \\
& \leq \sum_{k=1}^{n}\left(\sum_{(2 x)^{\alpha_{k-1}<p \leq(2 x)^{\alpha_{k}}}} \frac{1}{p}\right)\left(\sum_{\sqrt{2 x}<q \leq x /(2 x)^{\alpha_{k-1}}} \frac{1}{q}\right) \\
& =\sum_{k=1}^{n} \log \left(\frac{\alpha_{k}}{\alpha_{k-1}}\right) \log \left(2-2 \alpha_{k-1}\right)+o(1),
\end{aligned}
$$

as $x \rightarrow+\infty$. The desired bound follows by taking $n=10^{6}$. (The author performed the computation using the PARI/GP computer algebra system.)

For all $x, y \geq 0$, let $\Phi(x, y)$ be the number of positive integers not exceeding $x$ and having all their prime factors greater than $y$. We will make use of the following estimate for $\Phi(x, y)$.

Theorem 2.5. We have

$$
\Phi(x, y)=\frac{e^{-\gamma} x}{\log y} \cdot\left(1+O\left(\frac{1}{\log y}\right)\right)
$$

for all $x \geq 2$ and $2 \leq y \leq \exp (\log x / 10 \log \log x)$, where $\gamma$ is the Euler-Mascheroni constant.

Proof. It follows from [11, Chapter I.1, Theorem 11] and [11, Chapter I.4, Theorem 2].

\section{Proof of TheOrem 1.2}

Let $\mathcal{C}:=\mathbb{N} \backslash \mathcal{A}$ be the complement of $\mathcal{A}$. Clearly, it is enough to prove that $\mathcal{C}$ has lower density at least $\log 2+0.05551$.

For $x>0$, let $\mathcal{C}_{1}$ be the set of positive integers $n \leq x$ having a prime factor greater than $\sqrt{2 x}$. We shall prove that $\mathcal{C}_{1} \subseteq \mathcal{C}(x)$ and $\# \mathcal{C}_{1} \geq(\log 2+o(1)) \cdot x$, as $x \rightarrow+\infty$. To this aim, we proceed exactly as in the proof of Theorem 1.1. We include here the reasonings for the sake of completeness, and also to motivate better the rest of the proof. If $n \in \mathcal{C}_{1}$, then we can write $n=d p$ for a prime number $p>\sqrt{2 x}$ and a positive integer $d$ satisfying

$$
d \leq \frac{x}{p}<\frac{x}{\sqrt{2 x}}=\frac{1}{2} \sqrt{2 x}<\frac{p}{2} .
$$

Hence, by Lemma 2.1, $p$ does not divide $\left(\begin{array}{c}2 n \\ n\end{array}\right)$, so that $n \in \mathcal{C}$, and thus $\mathcal{C}_{1} \subseteq \mathcal{C}(x)$.

Moreover, for each prime number $p \in] \sqrt{2 x}, x]$, the number of possible $n=d p \in \mathcal{C}$ is at least $x / p-1$. Note also that, since $p>\sqrt{2 x}$, to each $n \in \mathcal{C}$ it corresponds exactly one $p$. Therefore,

$$
\# \mathcal{C}_{1} \geq \sum_{\sqrt{2 x}<p \leq x}\left(\frac{x}{p}-1\right) \geq x \cdot \sum_{\sqrt{2 x}<p \leq x} \frac{1}{p}-\pi(x)=(\log 2+o(1)) \cdot x,
$$

as $x \rightarrow+\infty$, where we used Theorem 2.3 and the well-known fact that $\pi(x)=o(x)$.

Now let $\mathcal{C}_{2}$ be the set of positive integers $n \leq x$ such that $n=m p$, where $\left.\left.p \in\right] \sqrt[3]{2 x}, \sqrt{2 x}\right]$ is a prime number, and $m$ is a positive integer having no prime factor greater than $\sqrt{2 x}$ and satisfying $(m \bmod p)<p / 2$. If $n \in \mathcal{C}_{2}$, then writing $n=m p$ as before we have

$$
m \leq \frac{x}{p}<\frac{x}{\sqrt[3]{2 x}}=\frac{1}{2}(2 x)^{2 / 3}<\frac{1}{2} p^{2},
$$

which, together with $(m \bmod p)<p / 2$, implies that $m=d_{1}+d_{2} p$ for some nonnegative integers $d_{1}, d_{2}<p / 2$. Hence, by Lemma $2.1, p$ does not divide $\left(\begin{array}{c}2 n \\ n\end{array}\right)$, so that $n \in \mathcal{C}$, and thus $\mathcal{C}_{2} \subseteq \mathcal{C}(x)$. 
At this point, we want to prove a lower bound for $\# \mathcal{C}_{2}$. For each prime number $p \in$ ]$\sqrt[3]{2 x}, \sqrt{2 x}]$, the number of positive integers $m \leq x / p$ such that $(\bmod \bmod )<p / 2$ is at least

$$
\left(\frac{x}{p^{2}}-1\right) \cdot \frac{p}{2}-1 \geq \frac{x}{2 p}-p
$$

Furthermore, the number of positive integers $m \leq x / p$ such that $m$ has a prime factor $q>\sqrt{2 x}$ is at most

$$
\sum_{\sqrt{2 x}<q \leq x / p} \frac{x}{p q} .
$$

Therefore, the number of positive integers $m$ such that $m p \in \mathcal{C}_{2}$ is at least

$$
\frac{x}{2 p}-p-\sum_{\sqrt{2 x}<q \leq x / p} \frac{x}{p q} .
$$

Now summing (4) over all prime numbers $p \in] \sqrt[3]{2 x}, \sqrt{2 x}]$ we get

$$
\begin{aligned}
\sum_{\sqrt[3]{2 x}<p \leq \sqrt{2 x}} & \left(\frac{x}{2 p}-p-\sum_{\sqrt{2 x}<q \leq x / p} \frac{x}{p q}\right) \\
& \geq \sum_{\sqrt[3]{2 x}<p \leq \sqrt{2 x}}\left(\frac{1}{2 p}-\sum_{\sqrt{2 x}<q \leq x / p} \frac{1}{p q}\right) \cdot x-\pi(\sqrt{2 x}) \cdot \sqrt{2 x} \\
& >\left(\frac{1}{2} \log \frac{3}{2}-0.06502+o(1)\right) \cdot x,
\end{aligned}
$$

as $x \rightarrow+\infty$, where we applied Theorem 2.3 and Lemma 2.4. However, note that (5) is not a lower bound for $\# \mathcal{C}_{2}$, since some $n \in \mathcal{C}_{2}$ could be written as $n=m p$ for two (and no more than two, since $p>\sqrt[3]{2 x}$ ) different values of $p$. The number of those double-counted $n$ 's is at most

$$
\sum_{\sqrt[3]{2 x}<p<q \leq \sqrt{2 x}} \frac{x}{p q}<\frac{x}{2} \cdot\left(\sum_{\sqrt[3]{2 x}<p \leq \sqrt{2 x}} \frac{1}{p}\right)^{2}=\left(\frac{1}{2}\left(\log \frac{3}{2}\right)^{2}+o(1)\right) \cdot x,
$$

as $x \rightarrow+\infty$, thanks again to Theorem 2.3. Hence,

$$
\# \mathcal{C}_{2}>\left(\frac{1}{2} \log \frac{3}{2}-0.06502-\frac{1}{2}\left(\log \frac{3}{2}\right)^{2}+o(1)\right) \cdot x>(0.05551+o(1)) \cdot x,
$$

as $x \rightarrow+\infty$.

At this point, since $\mathcal{C}_{1} \cap \mathcal{C}_{2}=\varnothing$ and $\mathcal{C}_{1} \cup \mathcal{C}_{2} \subseteq \mathcal{C}(x)$, by (3) and (6) we get

$$
\# \mathcal{C}(x) \geq \# \mathcal{C}_{1}+\# \mathcal{C}_{2}>(\log 2+0.05551+o(1)) \cdot x,
$$

as $x \rightarrow+\infty$, and the proof is complete.

\section{Proof of Theorem 1.3}

Suppose $x \geq 2$ is sufficiently large, and put $y:=\exp (\sqrt{\log x} / 4)$. We split $\mathcal{B}$ into two subsets:

$$
\begin{aligned}
& \mathcal{B}_{1}:=\{n \in \mathcal{B}: p \mid n \text { for some prime number } p \leq y\}, \\
& \mathcal{B}_{2}:=\mathcal{B} \backslash \mathcal{B}_{1} .
\end{aligned}
$$

If $n \in \mathcal{B}_{1}(x)$, then $n=m p$ for a prime number $p \leq y$ and a positive integer $m$. Moreover, since $n$ and $\left(\begin{array}{c}2 n \\ n\end{array}\right)$ are relatively prime, $p$ does not divide $\left(\begin{array}{c}2 n \\ n\end{array}\right)$, which in turn, by Lemma 2.1, implies that $p$ does not divide $\left(\begin{array}{c}2 m \\ m\end{array}\right)$. Now Lemma 2.2 tell us that for any prime number $p \leq y$ there 
are at most $p(x / p)^{\theta_{p}}$ positive integers $m \leq x / p$ such that $p$ does not divide $\left(\begin{array}{c}2 m \\ m\end{array}\right)$. Therefore,

$$
\begin{aligned}
\# \mathcal{B}_{1}(x) & \leq \sum_{p \leq y} p\left(\frac{x}{p}\right)^{\theta_{p}} \leq \sum_{p \leq y} p\left(\frac{x}{p}\right)^{1-1 /(4 \log p)} \ll x^{1-1 /(4 \log y)} \cdot y \\
& =x \cdot \exp \left(-\frac{\log x}{4 \log y}+\log y\right) \ll \frac{x}{\sqrt{\log x}},
\end{aligned}
$$

where we also used the inequality

$$
\theta_{p}=1-\frac{\log (2 p /(p+1))}{\log p}<1-\frac{1}{4 \log p} .
$$

On the other hand, thanks to Theorem 2.5, we have

$$
\# \mathcal{B}_{2}(x) \leq \Phi(x, y) \ll \frac{x}{\log y} \ll \frac{x}{\sqrt{\log x}} .
$$

Hence, putting together (7) and (8), we get

$$
\# \mathcal{B}(x)=\# \mathcal{B}_{1}(x)+\# \mathcal{B}_{2}(x) \ll \frac{x}{\sqrt{\log x}},
$$

as desired.

Acknowledgements. The author thanks the anonymous referee for a suggestion which improves considerably the bound of Lemma 2.4.

\section{REFERENCES}

1. J. J. Alba González, F. Luca, C. Pomerance, and I. E. Shparlinski, On numbers $n$ dividing the $n$th term of a linear recurrence, Proc. Edinb. Math. Soc. (2) 55 (2012), no. 2, 271-289.

2. R. André-Jeannin, Divisibility of generalized Fibonacci and Lucas numbers by their subscripts, Fibonacci Quart. 29 (1991), no. 4, 364-366.

3. A. S. Chen, T. A. Gassert, and K. E. Stange, Index divisibility in dynamical sequences and cyclic orbits modulo $p$, https://arxiv.org/abs/1608.02177.

4. E. E. Kummer, Über die Ergänzungssätze zu den allgemeinen Reciprocitätsgesetzen, J. Reine Angew. Math. 44 (1852), 93-146.

5. F. Luca and E. Tron, The distribution of self-Fibonacci divisors, Advances in the theory of numbers, Fields Inst. Commun., vol. 77, Fields Inst. Res. Math. Sci., Toronto, ON, 2015, pp. 149-158.

6. C. Pomerance, Divisors of the middle binomial coefficient, Amer. Math. Monthly 122 (2015), no. 7, 636-644.

7. C. Sanna, On numbers $n$ dividing the nth term of a Lucas sequence, Int. J. Number Theory 13 (2017), no. 3, 725-734.

8. C. Sanna, On numbers $n$ relatively prime with the $n$th term of a linear recurrence, Bull. Malays. Math. Sci. Soc. (2017, online).

9. N. J. A. Sloane, The On-Line Encyclopedia of Integer Sequences, https://oeis.org, Sequence A014847.

10. L. Somer, Divisibility of terms in Lucas sequences by their subscripts, Applications of Fibonacci numbers, Vol. 5 (St. Andrews, 1992), Kluwer Acad. Publ., Dordrecht, 1993, pp. 515-525.

11. G. Tenenbaum, Introduction to analytic and probabilistic number theory, Cambridge Studies in Advanced Mathematics, vol. 46, Cambridge University Press, Cambridge, 1995.

12. M. Ulas and A. Schinzel, A note on Erdös-Straus and Erdös-Graham divisibility problems (with an appendix by Andrzej Schinzel), Int. J. Number Theory 9 (2013), no. 3, 583-599.

Università Degli Studi di Torino, Department of Mathematics, Turin, Italy

E-mail address: carlo.sanna.dev@gmail.com 